\title{
Technological Challenges for Future Probiotic Foods
}

\author{
Meekha Mary Paul*, Anand, N., Arun Raj and S.N. Raghavendra \\ Department of Post-graduate Studies and Research in Food Science, St Aloysius College [Autonomous], Mangaluru, \\ India
}

*Corresponding author: meekhamary@gmail.com

Received: 07-03-2021

Revised: $27-05-2021$

Accepted: 17-06-2021

\begin{abstract}
In the modern era, people are interested in personal health and they take the food which is healthy and gives immunity to our body. Most people prefer functional foods which contain probiotics or live microbes. The market for probiotics has grown as demand for food and drinks, as well as dietary supplements and health awareness, has expanded. Between 2021 and 2027, the probiotics market is predicted to increase at an annual pace of $8.7 \%$, surpassing USD 4.30 billion. New product development with probiotics as the key supplement in it can also increase the growth of probiotics in the functional food markets.

Generally Recognized as Safe (GRAS) probiotic strains must be used in the production and the strains used should have stability and viability. Probiotic bacteria which have acid and heat tolerance and could withstand the conditions in the gastro intestine must be selected in the market. Before reaching the consumer, the manufacturer must ensure that all technological requirements have been met and that probiotics will survive throughout the product's production and storage. They must be acid, bile, and heat resistant, as well as viable and survive in the gastrointestinal environment. The most used delivery system of probiotics are dairy products but ice creams, chocolates, and certain beverages could also be used as carriers.

The future of the probiotic industry depends on the finding and implementation of new techniques which could increase the viability and shelf-life of the probiotic bacteria in our food. RBGR methodology is used to identify the heat-tolerant strains. Microencapsulation is a cutting-edge technology that solves the challenge of heat-tolerant strains surviving in severe environments. Novel production of probiotics in different foods and pharmaceuticals will provide new possibilities and new technologies. And hence maintaining low-cost productivity will be a great challenge with the new technologies being introduced.
\end{abstract}

Keywords: Probiotics, RBGR, immunity, gastro, intestine, bacteria, healthy

Probiotics are defined as "a live microbial food supplement that benefits the host by improving the intestinal microbial balance," as well as "living bacteria that exert health effects beyond the basic nutrition when taken in specific numbers". In fermented dairy products, cocktails of various bacteria, particularly Lactobacillus and Streptococcus species, have long been utilised to improve human health (Girardin et al. 2011).

Probiotics account for 65 percent of the global functional food business, which is predicted to be worth more than 75 billion dollars. The most defining active components of probiotic supplements are lactic acid bacteria such as bifidobacteria, lactobacilli, and enterococci. Probiotics have a variety of health claims, including the maintenance of normal/healthy intestinal flora and protection against infections,

How to cite this article: Paul, M.M., Anand, N., Raj, A. and Raghavendra, S.N. (2021). Technological Challenges for Future Probiotic Foods. Intl. J. Ferment. Food, 10(1): 35-50.

Source of Support: None; Conflict of Interest: None (क) 9 
relief of lactose intolerance, and immune system activation. Probiotics are bacteria strains that have been proved to offer health advantages and can be found in big amounts in foods. (Williams et al. 2010).

The adult human gastrointestinal system has a total mucosal surface area of up to $300 \mathrm{~m}^{2}$, making it the main bodily area that interacts with the environment. The gastrointestinal system is the body's largest lymphoid or immunological organ, thanks to gutassociated lymphoid tissue (GALT). According to estimates, the small bowel contains roughly 1010 immunoglobulin-producing cells per meter, accounting for nearly $80 \%$ of all immunoglobulinproducing cells in the body (Holzapfel et al. 2002).

In this context, probiotic cultures must be genetically characterised in order to clearly describe their contributions to the gut microbiota and, ultimately, to find the genotypes that control any distinctive and beneficial features. Strain selection and differentiation based on the genetic complement and programming of a potential probiotic becomes possible. Correlating major probiotic features with known genotypes and regulatory elements that are expected to influence functionality and beneficial effects in vivo will be critical to the field's advancement in the future.

\section{Selection and Production of Probiotics}

In the last five years, the probiotic sector has expanded with a slew of new cultures, each promising a different set of benefits. Lists of functional features and in vivo profit are now commonplace in any probiotics research. Companies seeking to develop a solid health settlement that will market their particular probiotics continue to prioritise scientifically verified health claims. The scientific community faces a bigger problem in determining cause and effect linkages for a wide range of potential and currently studied probiotic species and strain combinations. It will be necessary to create a platform that has critical information about the physiology and genetics of candidate strains that is relevant to their intestinal roles, functional activities, and interactions with other resident microflora for rational selection and design of probiotics, which will entail a platform of crucial information about the physiology and genetics of candidate strains relevant to their intestinal roles, functional activities, and interactions with other inhabitant microflora (Klaenhammer et al. 1999).

To qualify as a possible candidate for further screening for probiotic properties and associated health benefits, safe transit through the stomach and sustained existence and colonisation in the intestinal system are crucial and main parameters (Kotzamanidis et al. 2010). Beneficial bacteria that will be consumed as probiotic strains will be exposed to a wide range of stresses throughout their mobility and establishment in the gastrointestinal tract. Probiotic bacteria must maintain viability in the small intestine when exposed to stomach acid, bile, and high osmolarity (Franz and Holzapfel 2011). Water, inorganic ions, hydrochloric acid (HCL), other inorganic ions, pepsinogen, mucus, polypeptides, and intrinsic components make up gastric juice. The small $\mathrm{pH}$ range of human gastric juice, which runs from 1.5 to 3.5 , is due to HCL, which is secreted by stomach parietal cells (Marieb and Hoehn 2010). However, it differs at different times, such as when you have a blank stomach when you have a meal, and when you have a meal after a meal. With this in mind, a probiotic bacterial strain ought to be able to survive gastric acid's $\mathrm{pH}$ stress. Acid tolerance of isolates is tested using these criteria at various $\mathrm{pH}$ levels (1.5 to 4.5 ) and time intervals ( 0 to 3 hours) to simulate human stomach passage.

Acid tolerant strains are those that can withstand $\mathrm{pH}$ stress for an extended period of time. In response to a meal stimulus, bile is produced by liver hepatocytes and secreted in the small intestine via the gall bladder. It is a complex mixture of organic (conjugated bile acids, often known as bile salts; glycine, etc.) and inorganic compounds with antibacterial activity, primarily by bacterial membrane disintegration (Hofmann and Eckmann 2006). Microbes are exposed to bile salts ( $\mathrm{Ox}$ bile) in varying concentrations ( 0.5 to 2.0 percent) with varying contact times ( 0 to $3 \mathrm{hr}$ ). Bile tolerant isolates are those that show tolerance to bile. The sight of bile salt hydrolases (BSH), a product of the bsh gene in bacteria, is thought to be responsible 
for tolerance to bile acids (Begley et al. 2006). The BSH gene's presence and expression have been linked to bile tolerance and detoxification, and it's being used as a criterion for probiotic strain selection (Patel et al. 2010).

BSH is also thought to help lower cholesterol levels in the blood (El-Shafie et al. 2009), however, it varies per strain. Probiotic strains must stick to the intestinal lining after safe passage through the small intestine, which is aided by various factors such as cell wall hydrophobicity, cell adhesion potential, and auto-aggregation. Lactobacilli's capacity to cling to the intestinal epithelial cell lining is one of their most essential characteristics, as well as one of the most crucial factors to consider when choosing probiotic strains. (Ouwehand et al. 1999). First and foremost, bacterial cell wall hydrophobicity is critical for bacterial binding. Due to the removal of water between two surfaces, a hydrophobic connection begins immediately after contact and can become stronger over time (Younes et al. 2012). The type and location of bacteria's hydrophobic surface components determine the stability of this contact.

The BATH or MATH (bacterial/ microbial adherence to hydrocarbons) systems proposed by Rosenberg (1984) and Geertsema-Doornbusch and co-workers (1993) systems are commonly used to measure bacterial hydrophobicity. Hydrophobic strains have higher adhesion to hydrocarbons, while hydrophilic strains have lower adherence to hydrocarbons. Higher bacterial hydrophobicity is directly related to their capacity to stick to surfaces (Pan et al. 2006). The ability of bacterial cells to self-assemble is also important for bacterial adherence to intestinal cells (Dunne et al. 2001). It describes bacterial cells' activity in interacting with them in a distracted manner, which is required for GIT colonisation (del Re et al. 2000). Beneficial effects of probiotic strains can be attributed to their contact with other microorganisms (anti-microbial activity), metabolic activity (antioxidative activity, EPS generation, and so on), or their participation in immunological regulation via various signaling pathways (anti-cancerous; Antiallergic, etc.).
Antimicrobial activity is regarded as a critical functional parameter for competitively suppressing (pathogen exclusion) pathogenic intestinal microflora by the production of organic acids, hydrogen peroxide, and bacteriocins, among other things, so rendering the host safe. Following a typical well diffusion assay, the antagonistic activity of various bacterial measures is determined against common enteric pathogens (E. coli; Salmonella sp., etc.). LABs are also known to have strong anti-oxidant properties, reducing the likelihood of reactive oxygen species (ROS) formation (Achuthan et al. 2012). The difference between ROS or free radical production and body antioxidant defense causes oxidative stress, which disrupts normal cellular functions and has been implicated in a number of clinical conditions (inflammatory bowel disease, atherosclerosis, myocardial infarction, stroke, and vascular dysfunctionality, Alzheimer's disease, etc). The antioxidative potential of contestant probiotics can be assessed by assessing their resistance to ROS (hydrogen peroxide, hydroxyl ions, and superoxide radicals) (Kullisaar et al. 2002); Superoxide dismutase activity (Achuthan et al. 2012); and Total antioxidative activity (Kullisaar et al. 2002). In bacteriahost interactions, the presence of S-layer proteins, exo-polysaccharides (EPS), and other cellular envelope mechanisms are critical. Adhesion factors have been found on the surface polysaccharides of the EPS and S-layers (Lebeer et al. 2010; Sanchez et al. 2012).

The use of new DNA and protein-based technologies is rapidly increasing, and traditional screening methods are likely to be phased out (Papadimitriou et al. 2015). Because in vitro investigations do not replicate the natural environment of the gastrointestinal tract, health benefits demonstrated by a probiotic strain in laboratory form should be confirmed in an animal model before being tested in humans. The health benefits of suggested probiotic candidates should be established in animal models before they are given the probiotic designation, according to $\mathrm{FAO} / \mathrm{WHO}$ recommendations. Omics technologies may potentially prove valuable in the 
follow-up investigation of probiotic candidate strains identified by current techniques in vitro and/or in vivo screening. Despite the fact that there are various probiotic products available for human use, there is much debate about their benefits. This is due in part to the overblown and unjustified claims made in the product's advertising and marketing. Many stories of their favorable health effects have been published, with only a smattering of scientific evidence to back them up. Furthermore, many of the microorganisms used in these products are ineffective and were not chosen for specific therapeutic characteristics or the ability to survive in the gastrointestinal tract.

When employing probiotic microorganisms in fermented foods like yogurt, several things must be considered. To achieve the desired advantages, probiotics must first be viable and available in high numbers at the time of consumption (Castro et al. 2012). Thus, at the moment of consumption, a probiotic dairy product should contain at least 6-7 $\log$ CFU.g-1 of viable probiotic bacteria and should be ingested in quantities greater than $100 \mathrm{~g}$ per day, or at least $9 \log$ CFU per day (Codex, 2003). The inclusion of prebiotic sources like inulin has also been found to help fermented milk products regain their stability and sensory characterisation (Agil et al., 2013). In addition, several supplements, such as whey and whey protein concentrate, have improved microbial viability, physicochemical properties, and sensory qualities of probiotic dairy products.

The following are the selection factors for choosing the right probiotic bacteria and strains:

(a) The bacteria in question must be reported in the copy.

(b) Actual evidence of their contribution to health must be provided.

(c) They must beable to colonise the gastrointestinal tract and play a regulatory role in the microbial balance in that area.

(d) They must be resistant to low $\mathrm{pH}$ values and bile salt in order to maintain their capability in gastrointestinal conditions. (e) They must have a natural antibiotic effect in order to avoid pathogen growth with their antimicrobial activity.

(f) They must be safe to consume and show no antibiotic resistance.

(g) They must be suitable for commercialization (Farrar and Bower 1967).

Furthermore, the rules recommend that the efficacy of an overseas probiotic be tested in an Indian population before it is introduced into the Indian market. As a result, the ICMR/DBT guideline has provided consumers with considerable protection against misleading probiotic manufacturers in the Indian market (Thakur et al. 2006).

\section{Probiotic Interaction with Starter Bacteria}

Probiotic starter cultures have a key function throughout fermentation, ripening, and storage, boosting their health-promoting characteristics, strengthening their microbiology, and improving their sensory quality, but they can also accelerate lipid oxidation, reducing shelf life. Individual or mixed microbe cultures are utilised to support and conduct fermentation in known quantities (Laranjo Marta 2019).

The interactions between lactic acid starter bacteria and probiotic bacteria were studied in order to find suitable strain combinations for producing probiotic dairy products. With a few exceptions, probiotic bacteria were found to be more suppressive towards lactic acid bacteria than vice versa, as the latter had no effect on the former's growth. The research of interactions using growth kinetics resulted in the establishment of four distinct behaviors between lactic acid starter and probiotic bacteria species (stimulation, delay, complete inhibition of growth, and no effects among them). When picking the optimal combination of strains to create a probiotic fermented dairy product, possible interactions among the strains should be considered to optimise their efficiency in the process and their survival in the products during cold storage (Vinderola G. 2022). 


\section{Lactic acid starter bacterium strain interactions: Assay for Well-Diffusion Agar}

A total of 24 lactic acid starter bacteria strains (eight strains each) from the species $S$. thermophilus, $L b$. delbrueckii subsp. bulgaricus and Lactococcus lactis were tested for interactions. Complete (a distinct absence of growth of the test strain surrounding the well) and weak (the presence of a partial inhibition halo around the well) inhibitions, as well as the absence of interaction, were discovered using this technology. The growth of $S$. thermophilus and $L b$. delbrueckii subsp. bulgaricus strains were unaffected by cell-free supernatants of Lc. lactis. Except for Lc. lactis 15-4 and SL3, which were softly removed by streptococci supernatants, a similar behavior was seen when Lb. delbrueckii subsp. bulgaricus and Lc. lactis strains developed in the presence of CFS of $S$. thermophilus. Except for $L b$. delbrueckii subsp. bulgaricus Ab1 and Gb1 on Lc. lactis SD5, 15-1, 15-4, 13-3, and SL3, and Lb. delbrueckii subsp. bulgaricus $\mathrm{Cb} 1$ on Lc. lactis 151, 15-4, 13-3, and SL3, Lactobacillus delbrueckii subsp. bulgaricus subsp. bulga (Vinderola G. 2022).

There was no effect in these circumstances. When $S$. thermophilus strains were matured in the presence of $L b$. delbrueckii subsp. bulgaricus supernatants, the results were the most varied. The absence of contact, as well as the findings of entire and weak inhibitions, were documented in this case. Lb. delbrueckii subsp. bulgaricus $\mathrm{Db} 1, \mathrm{~Eb} 3, \mathrm{~Eb} 4$, and $\mathrm{Hb} 2$ were the most inhibitory strains, while Lb. delbrueckii subsp.bulgaricus Ab1 and Gb1 showed a condensed inhibition scale. The most sensitive $S$. thermophilus strains, on the other hand, were 175 and DC1 (Vinderola G . 2022).

Starter cultures are an essential instrument for ensuring the safety of fermented products. Indeed, through mechanisms such as the synthesis of specific metabolites or competitive elimination, the bacteria that makeup starting cultures may limit or restrict the formation of spoilage and/or harmful populations. Thus, using starting cultures instead of chemical additives like nitrites and nitrates may lessen the demand for them (Laranjo Marta 2019).

\section{Technological Standard for Probiotics}

The technological aspect when a probiotic is selected is that it should be viable throughout the production and should have a shelf-life. They should be also suitable for large-scale production but their presence should not affect any properties of food like sensory or flavor. The probiotic strain must be GRAS-certified (generally recognised as safe). To control the viability and shelf life of the probiotics several technologies like microencapsulation techniques, usage of oxygenpermeable containers, and selection of particular gene strains are done. (P.B. S. Bhadoria 2011). Major technological standards in probiotics are acidifying ability, proteolytic ability, lipolytic ability, texturing ability, antimicrobial activity, flavoring ability.

\section{Acid And Bile Tolerance}

The selection of probiotic strains is done based on their technical performance and their tolerance to acid stresses. Initial evaluation of strains for use of probiotic culture is done using assays such as acid and bile transit. The main characteristic of probiotics after their ingestion is their acid tolerance as the stomach has low $\mathrm{pH}$.

In a study by Saima Inayat (2020), it is seen that the Lactobacillus acidophilus is more stable than Bifidobacterium bifidum and other yogurt culture bacteria. Lactobacillus acidophilus showed greater viability and less viability loss than the other cultures and Lactobacillus acidophilus can survive up to $4.0 \mathrm{pH}$ while Bifidobacterium bifidum is less acid-tolerant and less viable at low $\mathrm{pH}$. Lactobacillus and Bifidobacterium are commonly found in milk and other dairy products; they can also be used in other goods, such as fruit drinks. The $\mathrm{pH}$ of fruit juices varies between 2.5 and 3.7. Chou and Weimer (1999) isolated acid and bile-tolerant lactobacillus variants. Park, H.K (1995) reported acid-adapted Bifidobacterium breve having superior characteristics and could withstand environmental stresses like bile, hydrogen peroxide, and cold storage. So, acid-resistant strains which survive in food and host environment are used. 


\section{Heat Tolerance}

Thermotolerance is one of the required abilities for probiotics in fermentation. The heat-inducible thermal tolerance of the bacteria helps the Lactobacilli to adapt to the environment and increase the thermal tolerance (Teixeira 1994 and Gouesbet 2002). In an experiment of improved stress tolerance Desmond (2004) states that the chaperone proteins present are an important component of heat shock response and guide the proteins. They are known as heat shock proteins as they are formed when cells are exposed to heat and as a result, cause misfolding. According to Desmond (2001), heat stress improved the thermotolerance of lactococci and lactobacilli (Desmond 2001).

Ding and Shah (2007) investigated the acid, bile, and heat tolerance of eight probiotic strains, including Lactobacillus rhamnosus, Bifidobacterium longum, Lactobacillus rhamnosus, Lactobacillus rhamnosus, Lactobacillus rhamnosus, Lactobacillus rhamnosus, Lactobacillus rhamnosus, Lacto L. salivarius L. plantarum, L. plantarum, L. plantarum, L L. acidophilus B. paracasei B. lactis type $\mathrm{Bl}-\mathrm{O} 4$ and $\mathrm{B}$. lactis type $\mathrm{Bl}-\mathrm{O} 4 \mathrm{Bi}-07$ lactis is a kind of lactis. As a control, free probiotic strains were employed, and microencapsulation in an alginate mixture was used. The probiotic organism's heat tolerance was examined by exposing it to $65^{\circ} \mathrm{C}$ for 1 hour. After one hour of exposure, it was discovered that both microencapsulated and free probiotic bacteria had suffered equal viability decreases. Microencapsulation appears to provide probiotic organisms a better chance than those that are free.

\section{Oxygen Tolerance}

According to the reports and surveys of probiotic yogurts in the market it was seen that some with $L$. acidophilus and Bifidobacteria have had a decline in their viability while some others were reported with satisfactory viability (Iwana (1993) Shah $(1995,2000)$. Several parameters were identified, including acid and hydrogen peroxide produced by yogurt bacteria, culture conditions, type of strain concentrations of lactic and acetic acids, whey proteins, and interaction of probiotic species with yogurt starters (Kailasapathy and Supraidi (1996) Shah (2000)). The oxygen content in the product and oxygen permeability through the package are the two most serious issues that lead to viability loss (Klaver 1993).

The reactivity of $L$. acidophilus and Bifidobacteria towards oxygen is a major problem and several experiments and studies were done to evaluate this and find a problem. Then Talwalkar (2001) developed the Relative Bacterial Growth Ratio (RBGR) methodology, which allows for a quantitative evaluation of probiotic bacteria's oxygen tolerance. Using this technique, the manufacturers can easily differentiate the oxygen tolerance and helps in maintaining the viability of bacteria throughout. (Talwalkar 2001).

L. acidophilus is microaerophilic in nature and is more prone to oxygen than Bifidobacteria as they are anaerobic in nature, oxygen tolerance of Bifidobacteria is mainly strain-dependent. In research, Dave and Shah (1997) found that the amount of probiotic strain in yogurt, when kept at 35 days of storage, was 106CFU g-1 which is above the recommended count. While L. acidophilus was only found to be 103CFU g-1.

\section{Ability To Grow}

Dairy products are the most used type of carrier of probiotics, yogurts and cheese are the most frequently used products in the dairy industry as carriers. In researches (Stanton 1998 and Gardiner 1999) it says that the cheddar cheese shows a more protective and nutritious effect compared to the yogurt in the delivery of Lactobacilli and Enterococci. When milk or milk products are used to deliver the probiotic a buffer effect is produced, gastric juice or media simulating gastric juice helps in increasing $\mathrm{pH}$ and supports the survival of the Lactobacilli and Enterococci. Chocolate and ice creams can also be used as a carrier, Ice cream follows the same manufacturing process as frozen yogurt. Probiotics when induced in chocolate have a shelf life of about 2 years. Milk chocolate masses are used for their preparation where Lactobacillus casei and Lactobacillus paracasei are tolerant to $\mathrm{pH} 3.5$ are used. Cells can survive 3 hours of incubation at $\mathrm{pH}$ 
3.5 and neutral $\mathrm{pH}$ (6.5), whereas $80-100$ percent of cells can remain at $\mathrm{pH} 2.5$ (depending on the strain), and only $60 \%$ of cells can stay in the midst of $4 \%$ bile salts. LAB strains can exert inhibitory effects on pathogenic bacteria, both Gram-negative and Grampositive. (Hamyouni Rad 2015). Probiotics could also be used in fruit juices but it's a bit challenging because of low $\mathrm{pH}$ and the presence of other antimicrobial components which may result in the loss of viability of the probiotic bacteria. The L. paracasei NFBC 338 strain is the intestinal origin and has high acid and bile tolerance and it and is compatible with other types like cheese manufacture (Gardiner 2000).

\section{Manufacturing of Non-Dairy Probiotics}

The use of probiotics as a supplement for animals and humans has increased growth and probiotics have become one of the important supplements in day-to-day life. The most commonly consumed probiotics are Bifidobacteria which are used in food supplements and Lactobacillus spp. which produce lactase which helps in breaking down milk sugar. At present, dairy-based probiotics stand over the nondairy probiotic supplements but some of the people who are lactose intolerant, having an allergy, or vegan and vegetarian personnel prefer the use of non-dairybased food products. These help in preventing allergy in dairy products and by providing low cholesterolcontaining products which help keep their heart healthy or adiposity (Granato 2010; Stadnik and Dolatowski 2014; Vasudha and Mishra 2013).

Lactobacillus spp. and Bifidobacterium spp. have GRAS (Generally Recognized as Safe) designation as it's the most widely used in the food industry. Saccharomyces cerevisiae and $S$. boulardii also possess probiotic properties. Probiotics are incorporated according to their origin, external stress, viability they mainly depend on the $\mathrm{pH}$, storage temperature, oxygen level, and presence of respective microorganisms and inhibitors.

Nondairy probiotic foods mainly include soy, meat, cereals, fruits, and vegetables which are rich in antioxidants, minerals, proteins, carbohydrates, and other dietary fibres. Nondairy probiotics are mainly divided into cereal-based probiotics, fruit, and vegetable-based probiotics, soy-based probiotics, and meat-based probiotic foods.

\section{Fruit and Vegetable Based}

Probiotics can be incorporated into a different types of fruits and vegetables in form of juices, puree, pulp, beverages, dried fruit. Fruits and vegetables are rich in carbohydrates, dietary fibres, vitamins, polyphenols, and certain minerals and hence are referred to as healthy foods. (Sutton, 2007). According to several studies, aqueous extracts of kiwifruit and avocado had low cytotoxicity and higher anti-inflammatory activity in a Crohn's gene-specific assay (Sutton, 2007), and the non-aqueous solution of kiwifruit, avocado, and blueberry has higher cytotoxicity than the aqueous solution and has greater antiinflammatory properties than the aqueous solution. Micronutrients such as calcium, retinol, vitamin E, folate, nicotine acid, riboflavin, pantothenic acid, $\beta$ -carotene, and biotin on genetic damage and restore can be found in fruits and vegetables, so combining these micronutrients with the probiotic strain will be a future goal and a great challenge in probiotic juices, as it may provide more health benefits. Certain berries like blueberry, raspberry possess antimicrobial and pathogenic protection against food-borne pathogens (Ranadheera 2014).

The Health benefit of a probiotic is to overcome the unfavorable conditions in the gastrointestinal tract. A probiotic juice is acceptable when there is perfect maintenance of the cell count or viability of the cell and good shelf life. Low $\mathrm{pH}$ in fruit juices affects the viability of probiotic strains (Vasudha and Mishra, 2013). However, probiotic viability varies by strain; some strains, such as Lb. plantarum, Lb. acidophilus, and $L b$. casei, are acid-tolerant.

Juice always has low $\mathrm{pH}$ and high levels of organic acid; $\mathrm{pH}$ is an important factor that affects the viability of probiotic strain. In the probiotic strain, lactobacilli are tolerant to low $\mathrm{pH}$ ranging from 4.3 to 3.7 while Bifidobacterium does not survive even at a $\mathrm{pH}$ of 4.6. 


\section{Cereal Based Probiotics}

Cereals like wheat, maize, oats, barley have dietary fibres and many other physiological properties which help in nourishing the gut by appropriate carbohydrates as prebiotics, and they are also used as an encapsulation material to improve the viability of the prebiotics (Capozzi 2012). According to Lamsal and Faubion (2009), cereal and cereal-based food products include prebiotics, probiotics, or dietary fibres in our diet.

According to Katina (2007), the whole grain contains many phytochemicals including antioxidants, phytic acid, sterols, phenoliccompounds, and phytoestrogen. When the cereals are fermented, they provide an optimum $\mathrm{pH}$ condition and enzymatic degradation of phytate occurs which releases certain minerals like zinc, calcium, iron, and manganese (growth factor of Lactic acid bacteria). Strains of lactobacillus are complex microorganisms that use up the fermentable carbohydrates, nucleic acids, minerals, and B vitamins for their growth and hence fermentation of cereals helps in the growth of beneficial microbes and thus becomes a means of probiotic delivery. Consumption of whole cereal products, therefore, gives probiotics and other components present in grain (Lamsal and Faubion 2009).

Fermented cereal products have the effect of prebiotics, probiotics, and dietary fibres present in them while fermented dairy-based products are commonly associated with probiotics. The bran and germs contain many bioactive compounds and fibres which are healthy and can be used as probiotic carriers. Oats and barley are cereals that have a high hypocholesterolemic effect and high $\beta$ glucan and they help in decreasing the cholesterol level in blood and maintains a healthy heart.

\section{Meat Based Probiotics}

Meat is an integral part of human nutrition, raw, ripened and cured meat has been produced by fermentation method for a long time. During the fermentation process, several substances like lactic acid, pyruvic acid, alcohols, aldehydes, ketones, and carboxylic acids are formed and these determine the quality and storage of the meat (Hugas 1997). During the production of fermented meat, proteolysis occurs in meat which causes the muscle structure to break down, and proteins are degraded into small peptides and free amino acids and help to enhance the aroma, color, and taste of the final product (Stadnik and Dolatowski 2014). Further, some species like Bifidobacterium and Lactobacilli when added produce conjugated linoleic acid (CLA) which has many functional properties like modulating the immune and inflammatory response and cardiovascular diseases.

Fermented meat like sausage is uncooked or ready to eat and hence it is regarded as a good means for transfer of probiotics to intestines as probiotics are inserted in the protein and fat matrix. In a study for evaluation of probiotics, it was seen that the initial inoculum of $105 \mathrm{CFU} / \mathrm{g}$ of a lactobacillus strain after fermentation increased to $109 \mathrm{CFU} / \mathrm{g}$. In another evaluation of probiotic count in mutton sausage with L. acidophilus CCDM 476 and B. animalis 241a, higher viability of L. acidophilus was observed after 60 days of storage, but the texture and aroma of the mutton were improved with the addition of probiotics. (Rivera-Espinoza and Gallardo-Navarro 2013, Holko 2013). However, probiotic meat production has some technological problems that need to be resolved like the low water activity, low content of sugar, and their microflora. Probiotic strains used in the formulation must also be able to survive in meat microflora and should survive in the condition of fermented products.

\section{Soy-Based Probiotic}

Soy is from the soy plant. Soy can be processed into soy protein, soy milk, or soy fibres. They are used as a substitute for milk for lactose-intolerant people. Soy is suitable for the growth of Lactobacillus and Bifidobacterium and is a functional food as they could be treated for diseases like menopausal disorder, cancer, atherosclerosis. Soy milk products are also used to treat kidney stones as soy decreases the albumin content in urine which is administered with Lactobacillus plantarum. 
Consumption of soy oligosaccharide sometimes may cause bloating or cramping in order to avoid that fermentation of soy with probiotics like lactobacillus and bifidobacterium should be carried out. (Champagne 2010). The maximum probiotic effect of a strain depends on when the live bacteria present during consumption and fecal recovery is high, in an experiment with bifidobacterium it was found that the number of live bacteria during consumption and their fecal recovery was higher and thus survives acid and bile inside the stomach (Shimakawa 2003). In order to not lose the viability of the probiotic strain, the soy product should be freeze-dried, stored at a temperature of about $4^{\circ} \mathrm{C}$, and packed in a tetra pack, freeze-drying is more preferred than spray drying or other techniques (Wang 2006). In another study when the Lactobacillus acidophilus FTCC 0291 was maintained above $106 \mathrm{CFU} \mathrm{g}^{-1}$ for about 20 days in soy cream cheese, the probiotic strain used up the soy sugar and use up the soy proteins and produce lactic acid which reduces the $\mathrm{pH}$ and avoid the growth of other aerobes and anaerobes.

\section{Formulation of Probiotics Good Results in Starch Encapsulation of Lactic Acid Bacteria}

Probiotic foods have live supplementary microorganisms. It was good for the host, by regulating the gastrointestinal flora (Mershen Govender et al. 2013). The major probiotic organisms are Lactobacillus (such as L. casei, L. lactis, etc); Bifidobacterium;Bacillus;Streptococcus;Pediococcus; Enterococcus; and yeasts e.g. Saccharomyces cerevisiae and S. boulardii (H. Musikasang et al. 2009). The regular intake of probiotic foods can improve the immune system, lowers the cholesterol level, prevent gastrointestinal related diseases, improves gut health, reduces stress, and regulates lactose tolerance (Srikanjana Klayraung et al. 2009). To get the functional health benefits, the probiotic bacteria should contain at least a cell viability of $107 \mathrm{CFU} / \mathrm{g}$ or more till the end of product shelf-life (Haiteng Li et al. 2016). Good probiotic bacteria should have a set of technological characters such as, should be non-toxic, able to process the prebiotic and have heat, acid, oxygen, and bile tolerances. Probiotic strains should accomplish those characters to improve the efficiency in the host and should be safer to consume (Esther Sendra Nadal et al. 2010).

The Lactic Acid Bacteria (LAB) are major organisms added to probiotic foods. Because the lactic acid bacteria are much safer to consume, it has many health benefits and probiotic properties (Mehran Moradi et al. 2020). Lactic acid bacteria are commonly used in dairy and non-dairy food products such as yogurt, milk, buttermilk, cheese, sausages, chocolate, cereals products, and fruit juices (Andreas Feucht and Haesoo Kwak, 2013). The major two probiotic cultures are Lactobacillus and Bifidobacterium species (Mershen Govender et al. 2014). The health benefit of probiotic products is greater when using LAB cultures. LAB culture produces organic acids, functional biological ingredients, and exopolysaccharides. The exopolysaccharides are biopolymers produced majorly by LAB cultures. Exopolysaccharide has special biological and Physico-chemical properties (such as antimicrobial, antioxidant, and anti-cancer). Exopolysaccharides are preventing the growth of harmful organisms in food, enhances the health benefits, are used in industrial applications (e.g. as a gelling agent, stabilizer, additive, thickener, and emulsifier), and also used in food active packaging (in the production of edible films and coatings). The major disadvantage of exopolysaccharides is the low yield in LAB. Downstream processing and composition of culture medium are the major factors that affect the yield of exopolysaccharides in LAB culture (Mehran Moradi et al. 2020).

Lactic acid bacteria incorporated probiotic food consumption increasing day by day because of the numerous health benefits, but researchers found that the bioavailability of LAB incorporated probiotic foods is low. LAB cultures are very sensitive to low $\mathrm{pH}$. After oral consumption, lactic acid bacteria should enter the small intestine with a sufficient amount of viable count (viable count should be a minimum of $107 \mathrm{CFU} / \mathrm{g}$ for effective health benefit). While pass-through stomach lactic acid bacteria may die because of direct contact with low $\mathrm{pH}$ gastric acid (Li Mei et al. 2014). 
The effectiveness of probiotics is evaluated in processed foods products. Probiotic bacteria are very sensitive to environmental factors and it loses their cell viability when it undergoes processing and long time storage (Mary Ellen Sanders and Maria L. Marco, 2009). Food formulation, processing conditions, food component, type of organism used, heat effects, osmotic pressure, genus, $\mathrm{pH}$, oxygen content, duration of storage, temperature, and the gastrointestinal condition are the different factors that affect probiotic functional efficacy, survival, and physiological activity during manufacturing, storage, fermentation, and digestive system (Karthiyaini Damodharan et al. 2017).

In order to get functional probiotic bacteria, it has to be overcome several environmental factors. Microencapsulation of probiotic bacteria could be a better solution to overcome those issues. The encapsulation technique maintains the cell viability during the processing, storage and as well as ensures effective activity in the gut (Carolina González-Ferrero et al. 2020). Encapsulation is a method of cell immobilization (R. Dembczynski and T. Jankowski, 2002). Encapsulation is a process where the sensitive bioactive compound is separated from the adverse environment by closing up the cells within the encapsulating matrix or membrane. The encapsulation technique lowers the cell injury, enhance the cell resistance in growth condition and also improve the cell viability throughout the storage period (M.J. Martin et al. 2013). Encapsulation is a developed and successful method for protecting probiotic bacteria. Encapsulation techniques are mostly used in various industries such as sustained food active compounds, controlling the oxidative process, cover up the unpleasant flavors and odors, or act as a shell between the sensitive bioactive compounds and the adverse environment (Verica Manojlović et al. 2010). Encapsulation is done by various techniques such as spray-drying, emulsions, coacervation, electrospinning/electrospraying, impinging aerosol technique, and extrusions (Chaline Caren Coghetto et al. 2016). Different polymers are used as an excipient in encapsulation. Examples like starch and cellulose (Karthiyaini Damodharan et al. 2017).

Encapsulated probiotics lose cell viability during storage. When the relative humidity is high it increases the water content, leads to the activation of oxidative reactions and cell damage. Incorporation of prebiotics (e.g. inulin, high amylase corn starch powder) in encapsulation lowers the water activity and increases the stability during storage time and conditions (Karthiyaini Damodharan et al. 2017).

Encapsulation of lactic acid bacteria (Lactobacillus acidophilus) with and without polymers was tested. The result shows that using alginate/starch polymers maintains viability throughout storage and increases efficiency (T. Jankowski et al. 1997). The incorporation of Hi-maize starch in the encapsulation technique increases the survival of bacteria. Lactobacillus Plantarum $299 \mathrm{v}$ gives a higher probiotic count in the small intestine and improves potential biological efficiency in our body when it is encapsulated with maize starches. Various authors found that starch encapsulated lactic acid bacteria provides protection to the sensitive strains, preserve the number of viable bacteria, maintain the quality of cell viability during processing, storage, and as well as in the digestive system (Digambar Kavitake et al. 2018).

\section{Prebiotic as Probiotic Promoters}

Prebiotics are dietary fibers with a beneficial effect on the intestinal microflora. Prebiotics are rich in plant polysaccharides that contain complex compounds that are resistant to digestion (non-digestible by human digestive enzymes) in the intestine and these are also called non-digestible oligosaccharides.

Good prebiotic should be:

(h) Sensitive to fermentation by gut bacteria,

(i) Not affected by mammalian enzymes and gastric acid

(j) Able to improve the functional efficacy of beneficial organisms (Mengfei Peng et al. 2020).

Prebiotics are dietary fibers that contain short-chain carbohydrates and are beneficial to the host. Major 
Prebiotics applied in food products are lactulose, galactooligosaccharides, fructooligosaccharides, inulin and its hydrolysates, raffinose, mannose, lactulose, stachyose, maltooligosaccharides, and resistant starch (Carlos Ricardo Soccol et al. 2010). In wheat, tomatoes, asparagus, breast milk, and some other foods naturally contain prebiotic compounds, and to get a functional efficacy have to intake a large amount of these foods.

Commercial prebiotics are produced by:

(a) Segregation from plant source

(b) Hydrolysis of polysaccharides

(c) Microbial or enzymatic manufacture.

Most prebiotic is suitable for almost all type of processed food products, soluble in water and not able to detect after addition. Prebiotics are commonly added in dairy products, table spreads, butter-like products, dairy spreads, cream cheeses, processed cheeses, bakery products, and breakfast cereals (Sadeq Hasan Al-Sheraji et al. 2013).

After oral consumption, Prebiotics are pass through the mouth, stomach, small intestine to the lower gut and start fermenting in the colon. Prebiotics are effectively used by only some of the probiotic organisms. Prebiotic help in cell growth and improves the functional efficacy of probiotic bacteria. Prebiotics gives energy to the host by producing acids (Examples acetic and butyric acids). These acids are formed during fermentation. These shortchain fatty acids are also found in plant sources like onion, garlic, asparagus, artichoke, and many more (Sadeq Hasan Al-Sheraji et al. 2013). Prebiotics has many potential health benefits such as boost the immune system, increases the absorption of minerals in the body, regulating lipids in the body, lowers diabetes, reduces the risk of cancer, intestinal disease, cardiovascular disease, and non-insulin-dependent diabetes, lower cholesterol level and prevent acute gastroenteritis (Valéria Maria Caselato de Sousa et al. 2011).

Several studies show that prebiotics is chemically unstable during processing conditions like high temperature, low acidity, and browning reactions. Inulin loses stability (around $20 \%-100 \%$ ) when it was heated about 130 to 1950c for 1 hour. Hence, found that reduced growth of beneficial organisms. Fructo oligosaccharides start degrading in buffer solution during low $\mathrm{pH}$ and high temperature. Therefore, to obtain the desired health benefits, the chemical and functional stability of prebiotics should be maintained throughout processing and storage ( $\mathrm{J}$. Huebner et al. 2008).

Probiotics and prebiotics are may formulate in the foods in order to improve the balance of intestinal microorganisms. Synbiotic is a combination of both prebiotic and probiotic that beneficially affects the host by increasing the survival, viability, and the total number of live beneficial organisms. For example, in synbiotic chocolate mousse, where Lactobacillus paracasei (probiotic) and inulin (prebiotic) were added to increase the survival and functional efficacy of probiotics. These combinations also enhance the sensory properties of chocolate mousse (Silvia Marina González et al. 2014). Studies reported that synbiotic foods gave a greater effective immune health compare to probiotic or prebiotic alone (Mengfei Peng et al. 2019).

Many researchers found that synbiotic has better efficacy, fourvariousprobioticsstrainsareencapsulated with (Lactobacillus acidophilus, Lactobacillus casei, Bifidobacterium bifidum, and Bifidobacterium longum) prebiotic (fructooligosaccharides) as a coatingmaterial incorporated with sodium alginate and peptide tested for optimal processing conditions, performance, and survival rates. Results show that this combination of prebiotic, peptide, and alginate gives the highest survival to the probiotics (KUN-NAN CHEN et al. 2005). To increase the bifidobacteria in the intestine (bifidogenic effect), xylooligosaccharides need $2 \mathrm{~g} /$ day, fructooligosaccharides need $4 \mathrm{~g} / \mathrm{day}$, isomaltooligosaccharides need $10 \mathrm{~g} /$ day. Incorporation of prebiotic particularly inulin into the food product give greater cell viability, survival, and efficacy of probiotic organism (Sadeq Hasan Al-Sheraji et al. 2013). Incorporation of inulin (prebiotic) with L. casei (probiotic) to fermented yogurts and other dairy 
products enhances the flavor, texture, reduced yogurt syneresis. Several studies report the incorporation of inulin into the milk can enhance the co-cultures acidification rate, favored post-acidification, increase the bifidobacteria in the intestine (bifidogenic effect), and maintain cell viability during storage ( Esther Sendra Nada et al. 2010).

\section{CONCLUSION}

In recent trends, modern consumers give importance to healthier foods with reduced chemicals and act as preventive medicine. This increases the demand for probiotic, probiotic, and symbiotic food products. Probiotic organisms are beneficial to the host by decreasing the harmful organism in the gut and also have various health benefits. Prebiotics are dietary fibers beneficial for probiotic bacteria. Synbiotic is the incorporation of both prebiotic and probiotic. Probiotics are very sensitive and lose their bioavailability when are undergo various processing conditions, storage, and digestion. This is a major technological challenge. In this review, the encapsulation technique is the better solution to this issue. The encapsulation method there prevents the direct expose of the active compound and act as a protective layer. Encapsulated probiotic foods have higher bioavailability. Several studies have shown that encapsulated symbiotic foods have a greater functional activity with improved sensory properties.

\section{REFERENCES}

Abd El-Salam, Mohamed H., et al. 2011. "Preparation and properties of probiotic concentrated yoghurt (labneh) fortified with conjugated linoleic acid." International Journal of Food Science \& Technology 46(10): 2103-2110.

Agil, Rania, et al. 2013. "Lentils enhance probiotic growth in yogurt and provide added benefit of antioxidant protection." LWT-Food Science and Technology 50(1): 45-49.

Akshat Talwalkar and Kaila Kailasapathy. 1996. The Role of Oxygen in the Viability of Probiotic Bacteria with Reference to L. acidophilus and Bifidobacterium spp.

Aminlari, Ladan, et al. 2019. "Effect of probiotics Bacillus coagulans and Lactobacillus plantarum on lipid profile and feces bacteria of rats fed cholesterol-enriched diet." Probiotics and Antimicrobial Proteins, 11(4): 1163-1171.
Ayama, H., Sumpavapol, P. and Chanthachum, S. 2014. Effect of encapsulation of selected probiotic cell on survival in simulated gastrointestinal tract condition. Songklanakarin J. Sci. Technol., 36(3): 291-299.

Begley, Máire, Colin Hill, and Cormac GM Gahan. 2006. "Bile salt hydrolase activity in probiotics." Applied and Environmental Microbiology 72(3): 1729-1738.

Besseling-Van Der Vaart, I., Heath, M.D., Guagnini, F. and Kramer, M.F. 2016. In vitro evidence for efficacy in food intolerance for the multispecies probiotic formulation Ecologic ${ }^{\circledR}$ Tolerance (Syngut ${ }^{\mathrm{TM}}$ ). Beneficial Microbes, 7(1): 111-118.

Capozzi, V., Russo, P., Dueñas, M.T., López, P. and Spano, G. 2012. Lactic acid bacteria producing B-group vitamins: a great potential for functional cereals products. Applied Microbiology and Biotechnology, 96(6): 1383-1394.

Castro, Cristina, et al. 2012. "Bacterial cellulose produced by a new acid-resistant strain of Gluconacetobacter genus." Carbohydrate Polymers, 89(4): 1033-1037.

Champagne, C.P., Tompkins, T.A., Buckley, N.D. and Green-Johnson, J.M. 2010. Effect of fermentation by pure and mixed cultures of Streptococcus thermophilus and Lactobacillus helveticus on isoflavone and B-vitamin content of a fermented soy beverage. Food Microbiology, 27(7), 968-972.

Coghetto, C.C., Brinques, G.B. and Ayub, M.A.Z., 2016. Probiotics production and alternative encapsulation methodologies to improve their viabilities under adverse environmental conditions. International Journal of Food Sciences and Nutrition, 67(8): 929-943.

Damodharan, K., Palaniyandi, S.A., Yang, S.H. and Suh, J.W. 2017. Co-encapsulation of lactic acid bacteria and prebiotic with alginate-fenugreek gum-locust bean gum matrix: viability of encapsulated bacteria under simulated gastrointestinal condition and during storage time. Biotechnology and Bioprocess Engineering, 22(3): 265271.

Dave, R.I. and Shah, N.P. 1997. Effectiveness of ascorbic acid as an oxygen scavenger in improving viability of probiotic bacteria in yoghurts made with commercial starter cultures. International Dairy Journal, 7(6-7): 435-443.

Del Re, B. et al. 2000. "Adhesion, autoaggregation and hydrophobicity of 13 strains of Bifidobacterium longum." Letters in Applied Microbiology, 31(6): 438-442.

Dembczynski, R. and Jankowski, T. 2002. Growth characteristics and acidifying activity of Lactobacillus rhamnosus in alginate/starch liquid-core capsules. Enzyme and Microbial Technology, 31(1-2): 111-115.

Desmond, C., Stanton, C., Fitzgerald, G.F., Collins, K. and Paul Ross, R. 2001. Environmental adaptation of probiotic lactobacilli towards improvement of performance during spray drying. International Dairy Journal, 11(10): 801-808. 
Ding, W.K. and Shah, N.P. 2007. Acid, Bile, and Heat Tolerance of Free and Microencapsulated Probiotic Bacteria. Journal of Food Science, 72(9): M446-M450.

Doleyres, Y. and Lacroix, C.J.I.D.J. 2005. Technologies with free and immobilised cells for probiotic bifidobacteria production and protection. International Dairy Journal, 15(10): 973-988.

Donthidi, A.R., Tester, R.F. and Aidoo, K.E. 2010. Effect of lecithin and starch on alginate-encapsulated probiotic bacteria. Journal of Microencapsulation, 27(1): 67-77.

Dunne, C., O’Mahony, L., Murphy, L., Thornton, G., Morrissey, D., O'Halloran, S., ... and Collins, J.K. 2001. In vitro selection criteria for probiotic bacteria of human origin: correlation with in vivo findings. The American Journal of Clinical Nutrition, 73(2): 386s-392s.

Feucht, A. and Kwak, H.S., 2013. Microencapsulation of lactic acid bacteria (LAB). Food Science of Animal Resources, 33(2): pp.229-238.

Franz, Charles MAP, et al. 2011. "Enterococci as probiotics and their implications in food safety." International Journal of Food Microbiology, 151(2): 125-140.

Fredua-Agyeman, M. and Gaisford, S. 2015. “Comparative survival of commercial probiotic formulations: tests in biorelevant gastric fluids and real-time measurements using microcalorimetry." Beneficial Microbes, 6(1): 141-151.

Ganguly, Subha. 2013. "Supplementation of prebiotics, probiotics and acids on immunity in poultry feed: a brief review." World's Poultry Science Journal, 69(3): 639-648.

Gardiner, G.E., O'Sullivan, E., Kelly, J., Auty, M.A.E., Fitzgerald, G.F., Collins, J.K., ... Stanton, C. 2000. Comparative Survival Rates of Human-Derived Probiotic Lactobacillus paracasei and L. salivarius Strains during Heat Treatment and Spray Drying. Applied and Environmental Microbiology, 66(6): 2605-2612.

Gardiner, G.E., Ross, R.P., Wallace, J.M., Scanlan, F.P., Jägers, P.P.J.M., Fitzgerald, G.F. ... Stanton, C. 1999. Influence of a Probiotic Adjunct Culture of Enterococcus faeciumon the Quality of Cheddar Cheese. Journal of Agricultural and Food Chemistry.

Gardiner, G., Ross, R.P., Collins, J.K., Fitzgerald, G. and Stanton, C. 1998. Development of a Probiotic Cheddar Cheese Containing Human-Derived Lactobacillus paracasei Strains. Applied and Environmental Microbiology, 64(6): 21922199.

Girardin, Marc, and Ernest G. Seidman. 2011. “Indications for the use of probiotics in gastrointestinal diseases." Digestive Diseases, 29(6): 574-587.

González-Ferrero, C., Irache, J.M., Marín-Calvo, B., OrtizRomero, L., Virto-Resano, R. and González-Navarro, C.J., 2020. Encapsulation of probiotics in soybean protein-based microparticles preserves viable cell concentration in foods all along the production and storage processes. Journal of Microencapsulation, 37(3): 242-253.

Gouesbet, G., Jan, G. and Boyaval, P. 2002. Two-Dimensional Electrophoresis Study of Lactobacillus delbrueckii subsp. bulgaricus Thermotolerance. Applied and Environmental Microbiology, 68(3): 1055-1063.

Govender, M., Choonara, Y.E., Kumar, P., du Toit, L.C., van Vuuren, S. and Pillay, V. 2014. A review of the advancements in probiotic delivery: Conventional vs. non-conventional formulations for intestinal flora supplementation. Aaps. Pharm. Sci. Tech., 15(1): 29-43.

Granato, D., G.F. Branco, F. Nazzaro, A. G. Cruz, and J. A. F. Faria. 2010. Functional foods and nondairy probiotic food development: Trends, concepts, and products. Comprehensive Reviews in Food Science and Food Safety, 9: 292-302.

Hofmann, Alan F. and Lars Eckmann. 2006. "How bile acids confer gut mucosal protection against bacteria." Proceedings of the National Academy of Sciences, 103(12): 4333-4334.

Holko, I., Hrabě, J., Šalaková, A. and Rada, V. 2013. The substitution of a traditional starter culture in mutton fermented sausages by Lactobacillus acidophilus and Bifidobacterium animalis. Meat Science, 94(3): 275-279.

Holzapfel, Wilhelm H. and Ulrich Schillinger. 2002. "Introduction to pre-and probiotics." Food Research International, 35(2-3): 109-116.

Homayouni Rad, Mehrban Roudbaneh, M., Ghasemnezhad Tabrizian, V., Javadi, M., Harati, N., Hamayouni Rad, H., and Kasaie, Z. (2015) Chocolate as probiotic food.

Hood, S.K. and Zoitola, E.A. 1988. Effect of Low pH on the Ability of Lactobacillus acidophilus to Survive and Adhere to Human Intestinal Cells. Journal of Food Science, 53(5): 1514-1516.

Hugas, M. and Monfort, J.M. 1997. Bacterial starter cultures for meat fermentation. Food Chemistry, 59(4).

Iwana, H., Masuda, H., Fujisawa, T., Suzuki, H. and Mitsuoka, T. 1993. Isolation and Identification of Bifidobacterium spp. in Commercial Yogurts Sold in Europe. Bifidobacteria and Microflora, 12(1): 39-45.

Jankowski, T., Zielinska, M. and Wysakowska, A. 1997. Encapsulation of lactic acid bacteria with alginate/starch capsules. Biotechnology Techniques, 11(1): 31-34.

Kailasapathy, K. 2006. Survival of free and encapsulated probiotic bacteria and their effect on the sensory properties of yoghurt. LWT-Food Science and Technology, 39(10): 12211227.

Katina, K., Liukkonen, K.-H., Kaukovirta-Norja, A., Adlercreutz, H., Heinonen, S.-M., Lampi, A.-M, Poutanen, K. (2007). Fermentation-induced changes in the nutritional value of native or germinated rye. Journal of Cereal Science, 46(3): 348-355. 
Kavitake, D., Kandasamy, S., Devi, P.B. and Shetty, P.H., 2018. Recent developments on encapsulation of lactic acid bacteria as potential starter culture in fermented foods-A review. Food Bioscience, 21: 34-44.

Keen, P.H., Slater, N.K. and Routh, A.F. 2012. Encapsulation of lactic acid bacteria in colloidosomes. Langmuir, 28(46): 16007-16014.

Klaenhammer, Todd R. and Martin J. Kullen. 1999. "Selection and design of probiotics." International Journal of Food Microbiology, 50(1-2): 45-57.

Klaver, F.A.M., Kingma, F. and Weerkamp, A.H. 1993. Growth and survival of bifidobacteria in milk. Neth Milk Dairy J., 47: 151-164.

Klayraung, S., Viernstein, H. and Okonogi, S. 2009. Development of tablets containing probiotics: Effects of formulation and processing parameters on bacterial viability. International Journal of Pharmaceutics, 370(1-2): pp.54-60.

Kotzamanidis, Charalambos, et al. 2010. "Evaluation of adhesion capacity, cell surface traits and immunomodulatory activity of presumptive probiotic Lactobacillus strains." International Journal of Food Microbiology 140(2-3): 154-163.

Kullisaar, Tiiu, et al. 2002. "Two antioxidative lactobacilli strains as promising probiotics." International Journal of Food Microbiology 72(3): 215-224.

Lacey, Derek C. et al. 2012. “Defining GM-CSF-and macrophage-CSF-dependent macrophage responses by in vitro models." The Journal of Immunology, 188(11): 57525765.

Lamsal, B.P. and Faubion, J.M. 2009. The beneficial use of cereal andcereal components in probiotic foods. Food Reviews International, 25(2): 103-14.

Laranjo, Marta, Maria Eduarda Potes, and Miguel Elias. 2019. "Role of starter cultures on the safety of fermented meat products." Frontiers in Microbiology, 10: 853.

Li, H., Turner, M.S. and Dhital, S. 2016. Encapsulation of Lactobacillus plantarum in porous maize starch. Lwt., 74: 542-549.

Li, S., Ma, Y., Ji, T., Sameen, D.E., Ahmed, S., Qin, W., Dai, J., Li, S. and Liu, Y. 2020. Cassava starch/carboxymethylcellulose edible films embedded with lactic acid bacteria to extend the shelf life of banana. Carbohydrate Polymers, 248: 116805.

Liong, M.-T., Easa, A. M., Lim, P.-T. and Kang, J.-Y. 2009. Survival, growth characteristics and bioactive potential of Lactobacillus acidophilusin a soy-based cream cheese. Journal of the Science of Food and Agriculture, 89(8): 1382-1391.

Manojlović, V., Nedović, V.A., Kailasapathy, K. and Zuidam, N.J. 2010. Encapsulation of probiotics for use in food products. In Encapsulation technologies for active food ingredients and food processing (pp. 269-302). Springer, New York, NY.
Martin, M.J., Lara-Villoslada, F., Ruiz, M.A. and Morales, M.E. 2013. Effect of unmodified starch on viability of alginateencapsulated Lactobacillus fermentum CECT5716. LWT-Food Science and Technology, 53(2): 480-486.

Mei, L., He, F., Zhou, R.Q., Wu, C.D., Liang, R., Xie, R., Ju, X.J., Wang, W. and Chu, L.Y. 2014. Novel intestinal-targeted Ca-alginate-based carrier for $\mathrm{pH}$-responsive protection and release of lactic acid bacteria. ACS Applied Materials $\mathcal{E}$ Interfaces, 6(8): 5962-5970.

Messaoudi, M., Lalonde, R., Violle, N., Javelot, H., Desor, D., Nejdi, A., Bisson, J.F., Rougeot, C., Pichelin, M., Cazaubiel, M. and Cazaubiel, J.M. 2011. Assessment of psychotropiclike properties of a probiotic formulation (Lactobacillus helveticus R0052 and Bifidobacterium longum R0175) in rats and human subjects. British Journal of Nutrition, 105(5): 755764.

Moradi, M., Guimarães, J.T. and Sahin, S. 2020. Current applications of exopolysaccharides from lactic acid bacteria in the development of food active edible packaging. Current Opinion in Food Science.

Moumita, S., Goderska, K., Johnson, E.M., Das, B., Indira, D., Yadav, R., Kumari, S. and Jayabalan, R. 2017. Evaluation of the viability of free and encapsulated lactic acid bacteria using in-vitro gastro intestinal model and survivability studies of synbiotic microcapsules in dry food matrix during storage. LWT., 77: 460-467.

Muhammad Bilal, Saima Inayat, Saqib Manzoor, Muhammad Imran, Mushtaq Hussain Lashariand Aizaz Hassan. 2020. Viability of Probiotics (Lactobacillus acidophilus and Bifidobacterium bifidum) in set type yogurt made from buffalo milk.

Musikasang, H., Tani, A., H-kittikun, A. and Maneerat, S. 2009. Probiotic potential of lactic acid bacteria isolated from chicken gastrointestinal digestive tract. World Journal of Microbiology and Biotechnology, 25(8): 1337-1345.

Nadal, E.S., Sayas-Barberá, E., Fernández-López, J. and PérezAlvarez, J.A. 2010. Food formulation to increase probiotic bacteria action or population. Bioactive Foods in Promoting Health, pp. 335-351.

Nakkarach, A. and Withayagiat, U. 2018. Comparison of synbiotic beverages produced from riceberry malt extract using selected free and encapsulated probiotic lactic acid bacteria. Agriculture and Natural Resources, 52(5): 467-476.

Nampoothiri, K.M. and Bindhumol, I. 2010. Exopolysaccharide production and prevention of syneresis in starch using encapsulated probiotic lactobacillus plantarum.

Nguyen, T.H., Kim, Y., Kim, J.S., Jeong, Y., Park, H.M., Kim, J.W., Kim, J.E., Kim, H., Paek, N.S. and Kang, C.H. 2020. Evaluating the cryoprotective encapsulation of the lactic acid bacteria in simulated gastrointestinal conditions. Biotechnology and Bioprocess Engineering, 25(2): 287-292. 
Ouwehand, Arthur C. et al. 1999. "Probiotics: mechanisms and established effects." International Dairy Journal, 9(1): 43-52.

Bhadoriaand, P.B.S. and Mahapatra, S.C. 2011. Prospects, Technological Aspects and Limitations of Probiotics - A Worldwide Review.

Teixeris, P., Castro, H. and Kirby, R. 1995. Inducible thermotolerance in Lactobacillus bulgaricus.

Papadimitriou, Konstantinos, et al. 2015. “Discovering probiotic microorganisms: in vitro, in vivo, genetic and omics approaches." Frontiers in Microbiology, 6: 58.

Patil, A., Disouza, J. and Pawar, S. 2019. Shelf-life stability of encapsulated lactic acid bacteria isolated from sheep milk thrived in different milk as natural media. Small Ruminant Research, 170: 19-25.

Patrignani, F., Burns, P., Serrazanetti, D., Vinderola, G., Reinheimer, J., Lanciotti, R. and Guerzoni, M.E. 2009. Suitability of high pressure-homogenized milk for the production of probiotic fermented milk containing Lactobacillus paracasei and Lactobacillus acidophilus. The Journal of Dairy Research, 76(1): 74.

Pereira, J.O., Soares, J., Sousa, S., Madureira, A.R., Gomes, A. and Pintado, M. 2016. Edible films as carrier for lactic acid bacteria. LWT., 73: 543-550.

Perricone, M., Bevilacqua, A., Altieri, C., Sinigaglia, M. and Corbo, M. 2015. Challenges for the Production of Probiotic Fruit Juices. Beverages, 1(2): 95-103.

Quinto, E.J., Jiménez, P., Caro, I., Tejero, J., Mateo, J. and Girbés, T. 2014. Probiotic lactic acid bacteria: a review. Food and Nutrition Sciences, 5(18): 1765.

Ross, R.P., Desmond, C., Fitzgerald, G.F. and Stanton, C. 2005. Overcoming the technological hurdles in the development of probiotic foods.

Rivera-Espinoza, Y. and Gallardo-Navarro, Y. 2010. Non-dairy probiotic products. Food Microbiology, 27(1): 1-11.

Rosenberg, Eugene, et al. "The role of microorganisms in coral health, disease and evolution." Nature Reviews Microbiology, 5(5): 355-362.

Sánchez, Borja, et al. "Toward improving technological and functional properties of probiotics in foods." Trends in Food Science \& Technology, 26(1): 56-63.

Sanders, M.E. and Marco, M.L. 2010. Food formats for effective delivery of probiotics.

Sanders, M.E., Klaenhammer, T.R., Ouwehand, A.C., Pot, B., Johansen, E., Heimbach, J.T., Marco, M.L., Tennilä, J., Ross, R.P., Franz, C. and Pagé, N. 2014. Effects of genetic, processing, or product formulation changes on efficacy and safety of probiotics. Annals of the New York Academy of Sciences, 1309(1): 1-18.

Sekhar, M.S., Unnikrishnan, M.K., Vijayanarayana, K., Rodrigues, G.S. and Mukhopadhyay, C. 2014. Topical application/formulation of probiotics: will it be a novel treatment approach for diabetic foot ulcer?. Medical Hypotheses, 82(1): 86-88.

Shah, N.P. 2000. Probiotic Bacteria: Selective Enumeration and Survival in Dairy Foods. Journal of Dairy Science.

Shimakawa, Y. 2003. Evaluation of Bifidobacterium breve strain Yakult-fermented soymilk as a probiotic food. International Journal of Food Microbiology, 81(2): 131-136.

Sievert, Dawn M. et al. 2013. “Antimicrobial-resistant pathogens associated with healthcare-associated infections: summary of data reported to the National Healthcare Safety Network at the Centers for Disease Control and Prevention, 20092010." Infection Control and Hospital Epidemiology, 34(1): $1-14$.

Soomro, A.H., Masud, T. and Anwaar, K., 2002. Role of lactic acid bacteria (LAB) in food preservation and human health-a review. Pakistan Journal of Nutrition, 1(1): 20-24.

Stadnik, J. and Dolatowski, Z.J. 2013. Effect of Inoculation with Probiotics and Ageing Time on Selected Functional Properties and Oxidation of Proteins in Dry-Cured Pork Loins. International Journal of Food Properties, 17(4): 866-876.

Stadnik, J. and Z. J. Dolatowski. 2014. Effect of inoculation with probioticsand ageing time on selected functional properties and oxidation of proteins in dry-cured pork loins. Int. J. Food Properties, 17(4): 866-76.

Sultana, K., Godward, G., Reynolds, N., Arumugaswamy, R., Peiris, P. and Kailasapathy, K. 2000. Encapsulation of probiotic bacteria with alginate-starch and evaluation of survival in simulated gastrointestinal conditions and in yoghurt. International Journal of Food Microbiology, 62(1-2): pp.47-55.

Sutton, K.H. 2007. Considerations for the successful development and launch of personalized nutrigenomic foods. Mutation Research/Fundamental and Molecular Mechanisms of Mutagenesis, 622(1-2): 117-121.

Talwalkar, A., Kailasapathy, K., Peiris, P. and Arumugaswamy, R. 2001. Application of RBGR - a simple way for screening of oxygen tolerance in probiotic bacteria. International Journal of Food Microbiology, 71(2-3): 245-248.

Thakur, Nishchal, Namita Rokana, and Harsh Panwar. 2016. "Probiotics: Selection criteria, safety and role in health and." Journal of Innovative Biology January, 3(1): 259-270.

Thangrongthong, S., Puttarat, N., Ladda, B., Itthisoponkul, T., Pinket, W., Kasemwong, K. and Taweechotipatr, M. 2020. Microencapsulation of probiotic Lactobacillus brevis ST69 producing GABA using alginate supplemented with nanocrystalline starch. Food Science and Biotechnology, 29(11): pp.1475-1482.

van Reenen, Carol A. and Leon MT Dicks. 2011. “Horizontal gene transfer amongst probiotic lactic acid bacteria and other intestinal microbiota: what are the possibilities? A review." Archives of Microbiology, 193(3): 157-168. 
Vasudha, S. and Mishra, H.N. 2013. Nondairy probiotic beverages. International Food Research 2.

Wang, Y.-C., Yu, R.-C. and Chou, C.-C. 2006. Antioxidative activities of soymilk fermented with lactic acid bacteria and Bifidobacteria. Food Microbiology, 23(2): 128-135.

Williams, Nancy Toedter. 2010. "Probiotics." American Journal of Health-System Pharmacy, 67(6): 449-458.
Younis, K., Ahmad, S. and Jahan, K. 2015. “Health benefits and application of prebiotics in foods." Journal of Food Processing E Technology, 6(4): 1.

Zhang, Zhongwang, et al. 2018. "Roles and applications of probiotic Lactobacillus strains." Applied Microbiology and Biotechnology, 102(19): 8135-8143. 